\title{
Fast atomic transport without vibrational heating
}

\author{
E. Torrontegui, ${ }^{1}$ S. Ibáñez, ${ }^{1}$ Xi Chen, ${ }^{1,2}$ A. Ruschhaupt,${ }^{3}$ D. Guéry-Odelin,${ }^{4}$ and J. G. Muga ${ }^{1,4,5}$ \\ ${ }^{1}$ Departamento de Química Física, Universidad del País Vasco, Apartado 644, E-48080 Bilbao, Spain \\ ${ }^{2}$ Department of Physics, Shanghai University, 200444 Shanghai, China \\ ${ }^{3}$ Institut für Theoretische Physik, Leibniz Universität Hannover, Appelstraße 2, D-30167 Hannover, Germany \\ ${ }^{4}$ Laboratoire Collisions Agrégats Réactivité, CNRS UMR 5589, IRSAMC, Université Paul Sabatier, 118 Route de Narbonne, \\ F-31062 Toulouse CEDEX 4, France \\ ${ }^{5}$ Max Planck Institute for the Physics of Complex Systems, Nöthnitzer Strasse 38, D-01187 Dresden, Germany
}

(Received 15 October 2010; published 31 January 2011)

\begin{abstract}
We use the dynamical invariants associated with the Hamiltonian of an atom in a one dimensional moving trap to inverse engineer the trap motion and perform fast atomic transport without final vibrational heating. The atom is driven nonadiabatically through a shortcut to the result of adiabatic, slow trap motion. For harmonic potentials this only requires designing appropriate trap trajectories, whereas perfect transport in anharmonic traps may be achieved by applying an extra field to compensate the forces in the rest frame of the trap. The results can be extended to atom stopping or launching. The limitations due to geometrical constraints, energies, and accelerations involved are analyzed along with the relation to previous approaches based on classical trajectories or "fast-forward" and "bang-bang" methods, which can be integrated in the invariant-based framework.
\end{abstract}

DOI: 10.1103/PhysRevA.83.013415

PACS number(s): 37.10.Gh, 03.65.Ca, 03.65.Nk

\section{INTRODUCTION}

A key element to attain an exhaustive control of states and dynamics of cold atoms and ions is their efficient transport by moving the confining trap. In spite of the broad span of conditions, heating mechanisms, transport distances from microns to tens of centimeters, transport times, and accelerations involved, there are some common elements and objectives that allow for a rather generic theoretical treatment as the one presented in this paper. Transport should ideally be lossless and fast and lead to a final state as close as possible ("faithful") to the initial one, up to global phase factors, in the frame of the transporting trap. The latter requirement is characterized in the most demanding applications as a high-fidelity condition or, more generally, as a no-heating or at least minimal heating condition; equivalently, it is characterized by the absence or minimization of vibrational excitations at the end of the transport. Note that reaching a faithful final state is not incompatible with some transient excitation in the instantaneous basis at intermediate times, i.e., the process does not have to be slow or, in the usual quantum mechanical jargon, "adiabatic," although slowness is certainly a simple way to avoid heating, at least under ideal conditions.

Efficient atom transport is a major goal for many applications, such as quantum information processing in multiplexed trap arrays [1,2] or quantum registers [3]; controlled translation from the production or cooling chamber to interaction or manipulation zones [4-6]; accurate control of interaction times and locations, e.g., in cavity QED experiments [7], quantum gates [8], or metrology [9]; and velocity control to launch [10] or stop atoms $[11,12]$.

Different approaches have been implemented. Neutral atoms have been transported as thermal atomic clouds $[6,13]$, condensates [14], or individually [15,16], using magnetic or optical traps. The magnetic traps can be translated by moving the coils mechanically [5], by time-varying currents in a lithographic conductor pattern [17], or on a conveyor belt with a chain of permanent magnets [18]. Optical traps can be used as optical tweezers whose focal point is translated by mechanically moving lenses $[4,19]$ or by traveling lattices (conveyor belts) made with two counterpropagating beams slightly detuned with respect one another [15,16,20]. There are also mixed magneto-optical approaches [6]. For ions, controlled time-dependent voltages have been used in linear-trap-based frequency standards [9] and, more recently, in quantum information applications using multisegmented Paul traps [21], an array of Penning traps [22], and also 2D configurations [23].

As mentioned, an obvious solution, at least in principle, to avoid spilling or heating of the atoms is to perform a sufficiently slow (adiabatic) transport. For some applications, however, this takes too long. In particular, since transport could occupy most of the operation time of realistic quantum information algorithms, "shuttling times" need to be minimized [2,21]. In addition, long times may be counterproductive in practice and induce overheating from coils or fluctuating fields and decoherence. In summary, there are good reasons to reduce the transport time, and, indeed, several theoretical and experimental works have studied ways to make fast transport also faithful [19,24-26].

Invariant-based inverse engineering is ideally suited to this end. The main aim of this paper is to set the basic invariant-based inverse engineering transport theory, analog to the one developed recently for trap expansions [27]. We shall also show that previous approaches for efficient transport [24,25] and some generalizations are embraced by it and point out the potential limitations of the method. In Sec. II we shall provide the main concepts and formulas of the time-dependent quadratic-in-momentum invariants relevant for transport problems. The two main reference cases are (i) rigid harmonic oscillator transport and (ii) transport on an arbitrary trap with force compensation. In Sec. III we explain and apply the inversion technique; this is compared in Sec. IV with an alternative "bang-bang" approach based 
on time segments of constant acceleration. Section V deals with practical limitations, and Sec. VI discusses the results and draws the conclusions.

\section{DYNAMICAL INVARIANTS}

In a seminal paper Lewis and Riesenfeld derived a simple relation between the solutions of the Schrödinger equation of a system with time-dependent Hamiltonian and the eigenstates of the corresponding invariants [28]. They paid special attention to the time-dependent harmonic oscillator and its invariants quadratic in position and momentum, following earlier work by Ermakov on the classical oscillator [29]. From a classical physics point of view, Lewis and Leach found the general form of the Hamiltonian compatible with invariants quadratic in momentum [30], including nonharmonic potentials. This is the result that interests us here, together with the corresponding quantum formulations by Leach for the harmonic oscillator [31,32] and by Dhara and Lawande for taking anharmonicity into account [33]. In this section we shall state the main concepts and equations and apply them to standard transport problems.

A 1D Hamiltonian with an invariant that is quadratic in momentum must have the form $H=p^{2} / 2 m+V(q, t){ }^{1}$ with $[30,33]$

$V(q, t)=-F(t) q+\frac{m}{2} \omega^{2}(t) q^{2}+\frac{1}{\rho(t)^{2}} U\left[\frac{q-\alpha(t)}{\rho(t)}\right]$.

$\rho, \alpha, \omega$, and $F$ are arbitrary functions of time that satisfy the auxiliary equations

$$
\begin{gathered}
\ddot{\rho}+\omega^{2}(t) \rho=\frac{\omega_{0}^{2}}{\rho^{3}}, \\
\ddot{\alpha}+\omega^{2}(t) \alpha=F(t) / m,
\end{gathered}
$$

with $\omega_{0}$ being constant. Their physical interpretation in the context of transport is detailed below. The dynamical invariant, up to a constant factor, is given by

$$
\begin{aligned}
I= & \frac{1}{2 m}[\rho(p-m \dot{\alpha})-m \dot{\rho}(q-\alpha)]^{2} \\
& +\frac{1}{2} m \omega_{0}^{2}\left(\frac{q-\alpha}{\rho}\right)^{2}+U\left(\frac{q-\alpha}{\rho}\right)
\end{aligned}
$$

and verifies

$$
\frac{d I}{d t} \equiv \frac{\partial I(t)}{\partial t}+\frac{1}{i \hbar}[I(t), H(t)]=0
$$

so that $\frac{d}{d t}\langle\psi(t)|I(t)| \psi(t)\rangle=0$ for any wave function $\psi(t)$ that evolves with $H . \psi(t)$ may be expanded in terms of constant coefficients $c_{n}$ and eigenvectors $\psi_{n}$ of $I$,

$$
\begin{gathered}
\psi(q, t)=\sum_{n} c_{n} e^{i \alpha_{n}} \psi_{n}(q, t), \\
I(t) \psi_{n}(q, t)=\lambda_{n} \psi_{n}(q, t),
\end{gathered}
$$

where the $\lambda_{n}$ are time-independent eigenvalues. We shall generally deal with $\psi_{n}$ normalized to 1 , but continuum,

\footnotetext{
${ }^{1}$ Following the usual practice, $q$ and $p$ will denote operators or numbers, and the context should clarify their meaning.
}

delta-normalized states are also possible. The phases $\alpha_{n}$ satisfy $[28,33]$

$$
\begin{gathered}
\hbar \frac{d \alpha_{n}}{d t}=\left\langle\psi_{n}\left|i \hbar \frac{\partial}{\partial t}-H\right| \psi_{n}\right\rangle, \\
\alpha_{n}=-\frac{1}{\hbar} \int_{0}^{t} d t^{\prime}\left(\frac{\lambda_{n}}{\rho^{2}}+\frac{m(\dot{\alpha} \rho-\alpha \dot{\rho})^{2}}{2 \rho^{2}}\right) .
\end{gathered}
$$

The $\psi_{n}$ are, in practice, obtained easily as [33]

$$
\psi_{n}(q, t)=e^{\frac{i m}{\hbar}\left[\dot{\rho} q^{2} / 2 \rho+(\dot{\alpha} \rho-\alpha \dot{\rho}) q / \rho\right]} \frac{1}{\rho^{1 / 2}} \phi_{n}(\underbrace{\frac{q-\alpha}{\rho}}_{=: \sigma})
$$

from the solutions $\phi_{n}(\sigma)$, normalized in $\sigma$ space, of the auxiliary stationary Schrödinger equation

$$
\left[-\frac{\hbar^{2}}{2 m} \frac{\partial^{2}}{\partial \sigma^{2}}+\frac{1}{2} m \omega_{0}^{2} \sigma^{2}+U(\sigma)\right] \phi_{n}=\lambda_{n} \phi_{n}
$$

Whereas trap expansions and contractions imply a timedependent $\rho$ function [27], a large family of transport problems may be described by taking

$$
\rho(t)=1, \quad \omega^{2}(t)=\omega_{0}^{2}
$$

so that the auxiliary Eq. (2) plays no role and only Eq. (3) is relevant. Except in the final discussion we shall assume that the conditions (12) hold from now on and consider in detail two main reference cases.

(i) Rigid harmonic oscillator driven by the "transport function" $q_{0}(t)$. Hereafter this case is referred to as "harmonic transport" for short. Suppose that a harmonic trap is moved from $q_{0}(0)$ at time $t=0$ to $d=q_{0}\left(t_{f}\right)$ at a time $t_{f}$. In Eq. (1) this case corresponds to

$$
F=m \omega_{0}^{2} q_{0}(t), \quad \omega(t)=\omega_{0}, \quad U=0 .
$$

Adding to $V$ the irrelevant time-dependent global term $m \omega_{0}^{2} q_{0}^{2} / 2$, which produces no force, the trap potential can be written as a moving harmonic oscillator $m \omega_{0}^{2}\left[q-q_{0}(t)\right]^{2} / 2$,

$$
H=p^{2} / 2 m+m \omega_{0}^{2}\left[q-q_{0}(t)\right]^{2} / 2,
$$

and $\alpha$ may be identified with a classical trajectory $q_{c}$ since Eq. (3) becomes

$$
\ddot{q}_{c}+\omega_{0}^{2}\left(q_{c}-q_{0}\right)=0 .
$$

The invariants and transport modes will depend on it. In this case, $\lambda_{n}=E_{n}=(n+1 / 2) \hbar \omega_{0}$, and the transport mode $e^{i \alpha_{n}} \psi_{n}$ takes a physically transparent form,

$$
e^{i \alpha_{n}} \psi_{n}=e^{-\frac{i}{\hbar}\left[E_{n} t+\int_{0}^{t} \frac{m \dot{q}_{c}^{2}}{2} d t^{\prime}\right]} e^{i m \dot{q}_{c} q / \hbar} \phi_{n}\left(q-q_{c}\right)
$$

Efficient transport will be engineered in Sec. III by designing first an appropriate classical trajectory $q_{c}(t)$, from which the trap motion trajectory $q_{0}(t)$ is deduced via Eq. (15).

A variant of this case is vertical transport with a gravity force, so that $F=m \omega_{0}^{2} q_{0}-m g$ and Eq. (15) is modified to

$$
\ddot{q}_{c}+\omega_{0}^{2}\left(q_{c}-q_{0}\right)=-g .
$$


(ii) Arbitrary trap-driven transport with compensating force. Hereafter this case is referred to as the "compensatingforce approach" for short. Now, in Eq. (1),

$$
\begin{gathered}
\omega=\omega_{0}=0, \\
F=m \ddot{q}_{0} .
\end{gathered}
$$

In this case the trap potential $U\left[q-q_{0}(t)\right]$ is arbitrary; in particular, it could be harmonic, and it is rigidly displaced along $q_{0}(t)$, so $\alpha$ in Eq. (3) may be now identified with the transport function $q_{0}$. In addition to $U$, there is a timedependent linear potential term $-m q \ddot{q}_{0}$ in $H$,

$$
H=p^{2} / 2 m-m q \ddot{q}_{0}+U\left(q-q_{0}\right) .
$$

The corresponding force compensates exactly the inertial force due to the trap motion in the rest frame of the trap, so that the wave function in that frame is not modified up to a time-dependent global phase factor; see Appendix A. This Hamiltonian has been proposed by Masuda and Nakamura following a very different route, using a "fast-forward" scaling technique [25].

\section{INVERSE ENGINEERING METHOD}

The Lewis-Riesenfeld theory of invariants has been considered before in harmonic-oscillator-driven transport in the direct, rather than inverse, way by setting the transport function $q_{0}$ and analyzing the final heating, in particular, in adiabatic or quasiadiabatic regimes [2]. We shall use it instead as the basis for an inverse engineering approach, including also nonharmonic driving. The two main cases discussed above require different implementations. In both cases we shall assume that $q_{0}$ is displaced from 0 to $d$ in a time $t_{f}$.

\section{A. Harmonic transport}

In case (i) we may adopt, as in [27], an inverse engineering strategy by designing first the classical trajectory $q_{c}$ to assure that the transport modes coincide with the eigenvectors of the instantaneous Hamiltonian at initial and final times. This amounts to imposing the commutativity of $I(t)$ and $H(t)$ at $t=0$ and $t=t_{f}$, which can be achieved by setting [see Eq. (16)] the following boundary conditions:

$$
\begin{aligned}
& q_{c}(0)=q_{0}(0)=0 ; \quad \dot{q}_{c}(0)=0 ; \quad \ddot{q}_{c}(0)=0, \\
& q_{c}\left(t_{f}\right)=q_{0}\left(t_{f}\right)=d ; \quad \dot{q}_{c}\left(t_{f}\right)=0 ; \quad \ddot{q}_{c}\left(t_{f}\right)=0,
\end{aligned}
$$

with the last condition in these equations being determined by consistency with Eq. (15). $q_{c}(t)$ is then interpolated by assuming some flexible functional form, such as a polynomial, $q_{c}(t)=\sum_{n=0}^{5} \beta_{n} t^{n}$, where $\beta_{n}$ are found by solving the system of equations established by the boundary conditions. The resulting $q_{c}$ depends on time only trough the ratio $s=t / t_{f}$ and is directly proportional to $d$ :

$$
q_{c}(t) / d=10 s^{3}-15 s^{4}+6 s^{5} .
$$

Once $q_{c}$ is fixed, we get the trap trajectory from Eq. (15),

$$
q_{0}(t)=\ddot{q}_{c}(t) / \omega_{0}^{2}+q_{c}(t) .
$$

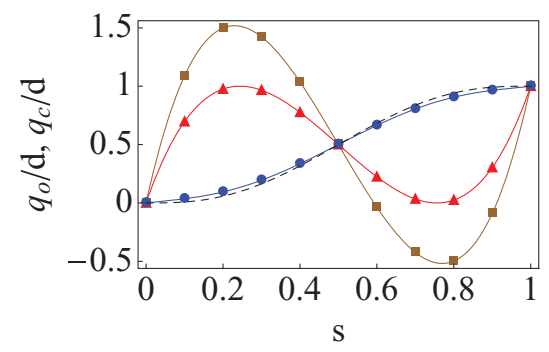

FIG. 1. (Color online) Trap trajectories $q_{0} / d$ versus $s=t / t_{f}$ for $t_{f}=12.57 / \omega_{0}=2 T_{0}$ (blue line with dots), $t_{f}=2.505 / \omega_{0}$ (the critical value; red line with triangles), and $t_{f}=2 / \omega_{0}$ (brown line with squares). For all three cases, $q_{c} / d$ is the dashed line, which is hardly distinguishable from $q_{0} / d$ for the slowest case, i.e., for $t_{f}=12.57 / \omega_{0}$.

This procedure is equivalent to the one followed by Murphy et al. [24], who used a Fourier sum as an interpolating function for $q_{c}$.

For short times $t_{f}$ (see Fig. 1), the corresponding trap trajectories $q_{0}(t)$ could exceed the interval $[0, d]$. For the polynomial ansatz (23) it occurs symmetrically at the lower and upper edges of the interval for $t_{f} \leqslant 2.505 / \omega_{0} \approx 0.4 T_{0}$, where $T_{0} \equiv 2 \pi / \omega_{0}$ is the oscillation period. This may or may not be a problem depending on the geometrical constraints of the experimental setting.

An interesting generalization is to consider boundary conditions for stopping atoms when their initial average velocity is known. Suppose that an atom gun or pulsed valve sends atoms with a specific average velocity $v_{0}$, as in coil-gun experiments with paramagnetic atoms [34,35] or a Stark decelerator for polar molecules [36-38]. The valve opening time is controlled, so a traveling harmonic trap wrapping the atoms can be turned on at time $t=0$ and moved along some trajectory $q_{0}(t)$ to stop them at a fixed distance $d$ in a specified time $t_{f}$. The final conditions may still be given by Eq. (22), but a different set of initial conditions is to be imposed:

$$
q_{c}(0)=0, \quad \dot{q}_{c}(0)=v_{0}, \quad \ddot{q}_{c}(0)=0 .
$$

In this case the $n$th initial transport mode at $t=0$ does not coincide with the $n$th stationary eigenstate of $H(0)$ but with its moving version, $e^{i m v_{0} q / \hbar} \phi_{n}(q) . \omega_{0}$ is in principle arbitrary, but it may be optimized by taking into account the spatial width of the incoming state. Its value also has an impact on the domain of the trajectory, as we shall see. The polynomial ansatz now gives

$$
\begin{aligned}
q_{c}(t)=d & {\left[3(2-a) s^{5}-(15-8 a) s^{4}-2(-5+3 a) s^{3}+a s\right], } \\
q_{0}(t)= & d\left\{3(2-a) s^{5}-(15-8 a) s^{4}\right. \\
& +\left[\frac{60(2-a)}{b^{2}}-2(-5+3 a)\right] s^{3}-12 \frac{(15-8 a)}{b^{2}} s^{2} \\
& \left.+\left[-12 \frac{(-5+3 a)}{b^{2}}+a\right] s\right\},
\end{aligned}
$$




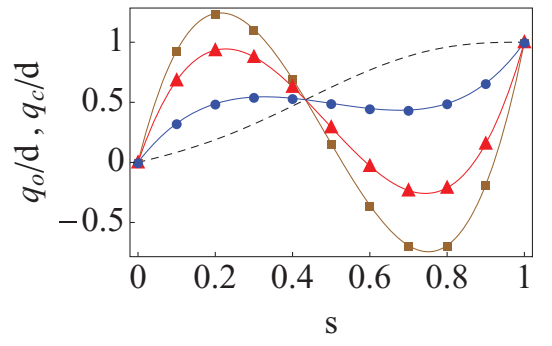

FIG. 2. (Color online) Three stopping trajectories: $a=0.8, b=$ $\omega_{0} t_{f}=1.6$, and $t_{f} / T_{0}=0.25$ (brown line with squares); $b=\omega_{0} t_{f}=$ 1.9 and $t_{f} / T_{0}=0.30$ (red line with triangles); and $b=\omega_{0} t_{f}=3.0$ and $t_{f} / T_{0}=0.477$ (blue line with circles). They all share the same $q_{c}(s) / d$ (black dashed line) given by Eq. (26).

where $s=t / t_{f}, a=v_{0} t_{f} / d$, and $b=\omega_{0} t_{f}=2 \pi t_{f} / T_{0}$. Some trajectories are shown in Fig. 2. The shaded regions in Fig. 3 correspond to the values of $a$ and $b / 2 \pi$ for which the trajectory $q_{0}(t)$ exceeds the domain $[0, d]$. Even though the details are now more complicated than for the rest-to-rest case, two simple general rules can be drawn: $q_{0}$ is never negative when $t_{f}>$ $T_{0}$ (the asymptotic threshold for large $a$ is at $b=6$ ), whereas if $a \geqslant 2.513$, there is always some $t$ in $\left(0, t_{f}\right)$ for which $q_{0}(t)>d$.

Launching or "catapulting" atoms at rest to end up with a chosen velocity $v_{0}$ [10] may be designed similarly by setting Eq. (21) for the initial conditions and setting the final boundary conditions as

$$
q_{c}\left(t_{f}\right)=d, \quad \dot{q}_{c}\left(t_{f}\right)=v_{0}, \quad \ddot{q}_{c}\left(t_{f}\right)=0 .
$$

A major practical concern in all these applications should be to keep the harmonic approximation valid. This may require an analysis of the actual potential in each specific case and of the excitations taking place along the nonadiabatic transport process. Without performing such detailed analysis, the feasibility of the approach for a given transport objective set by the pair $d, t_{f}$ can be estimated rather simply by comparing lower excitation bounds provided in Sec. V with the trap depth.

\section{B. Compensating-force approach}

In the compensating-force approach, case (ii), we may proceed similarly, but now the variable $\alpha$ of Eqs. (1) and (3) is directly $q_{0}$, so instead of fixing values of $q_{c}$ and its derivatives at the boundary times, we shall fix values of $q_{0}$ and its derivatives. For simplicity, we shall consider only a rest-to-rest scenario, but the generalizations are straightforward. The compensating potential $-m \ddot{q}_{0} q$ in the Hamiltonian (20) should vanish before and after transport since the trap remains at rest and $\ddot{q}_{0}=0$
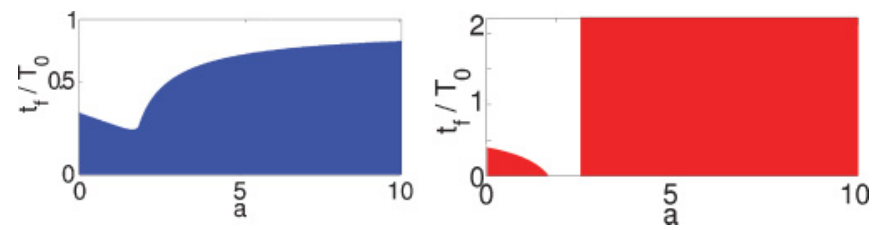

FIG. 3. (Color online) Stopping atoms. The shaded areas represent the values of $a=v_{0} t_{f} / d$ and $b / 2 \pi=t_{f} / T_{0}$ for which there is a $t$ in $\left(0, t_{f}\right)$ where (left) $q_{0}(t)<0$ or (right) $q_{0}(t)>d$. We have used the polynomial ansatz of Eqs. (26) and (27). for $t<0$ and $t>t_{f}$. To make the $\psi_{n}$ coincide, up to a global phase factor, with the eigenstates of the Hamiltonian before and after transport, $H=p^{2} / 2 m+U\left(q-q_{0}\right)$, at $t=0$ and $t=t_{f}$, we impose

$$
q_{0}(0)=\dot{q}_{0}(0)=0, \quad q_{0}\left(t_{f}\right)=d, \quad \dot{q}_{0}\left(t_{f}\right)=0 .
$$

We may also impose $\ddot{q}_{0}=0$ as a boundary condition at $t=0$ and $t_{f}$ to have a continuous $\ddot{q}_{0}(t)$, but at least formally, it is not strictly necessary. In practice, several experiments have been designed with (approximate) discontinuities in the trap acceleration $[16,19]$. We shall come back to this point in Sec. IV. Clearly, the implementation of the compensatingforce approach is subjected to different limitations from the ones applicable to the harmonic transport without compensation. The main problem now is not anharmonicity, which is included in the theory from the start by admitting an arbitrary $U$, but the feasibility of the compensating-force term. According to the mean-value theorem (see Sec. V) a lower bound for the maximum of the absolute value of $\ddot{q}_{0}$ is $2 d / t_{f}^{2}$.

\section{BANG-BANG ACCELERATION METHODS}

It should be clear from Sec. III A that there are infinitely many functions $q_{0}(t)$ that, for harmonic transport, lead to the ideal boundary conditions. A somewhat extreme case, which has, however, been implemented experimentally because of its relative simplicity, is to combine time segments with a constant acceleration [16,19]. The simplest trap trajectory of this type implies a constant positive acceleration $4 d / t_{f}^{2}$ from 0 to $t_{f} / 2$ and a deceleration $-4 d / t_{f}^{2}$ from $t_{f} / 2$ to $t_{f}$. The resulting $q_{0}$ is formed by two parabolas matched at $t_{f} / 2$ :

$$
q_{0} / d=\left\{\begin{array}{lc}
2 s^{2}, & 0<s<1 / 2, \\
4\left(s-\frac{s^{2}}{2}-\frac{1}{4}\right), & \frac{1}{2}<s<1 .
\end{array}\right.
$$

The corresponding velocity $\dot{q}_{0}$ increases linearly from 0 to $2 d / t_{f}$ and then decreases from there to 0 .

The classical trajectory $q_{c}$ satisfying Eq. (15) with Eq. (30) and the boundary conditions (21) at $t=0$ is given by

$$
q_{c}-q_{0}=\left\{\begin{array}{l}
\frac{-4 d}{\omega_{0}^{2} t_{f}^{2}}\left(1-\cos \omega_{0} t\right) \\
\frac{4 d}{\omega_{0}^{2} t_{f}^{2}}\left\{1+\cos \omega_{0} t-2 \cos \left[\omega_{0}\left(t-\frac{t_{f}}{2}\right)\right]\right\}
\end{array}\right.
$$

for the first and second time segments. From this result one can check that the boundary conditions at $t_{f}$ (22) are satisfied only for a discrete set of times $t_{f, N}=4 \pi N / \omega_{0}$, with $N \in \mathbb{N}$, i.e., for multiples of two oscillation periods. For all other times this scheme will heat the atoms. A perturbation theory analysis shows that, even for the selected discrete times, the bang-bang method is slightly less stable than the inverse invariant method, using the polynomial ansatz, with respect to an anharmonic perturbation of the transporting trap potential. The details are shown in Appendix B.

A variant of this method, using, e.g., the trap trajectory (30) and the compensating forces as in case (ii), may be appealing in practice because it is relatively simple to implement the compensating force, at least approximately, as a piecewise function. 


\section{TRANSIENT ENERGY EXCITATIONS}

Whereas, ideally, nonadiabatic faithful transport can be performed for arbitrary transport distances and times, in practice, the process could be limited, apart from the geometrical constraints discussed in Sec. IV, by the maximal transient excitation energies allowed to neglect the effect of anharmonicities of the actual potential in case (i) or by the difficulties to implement strong compensating forces in case (ii). We shall analyze these effects from the point of view of different bounds obtained for the average potential energy using the Euler-Lagrange equations and for the instantaneous potential energy and acceleration by means of the mean-value theorem.

\section{A. Quasioptimal trajectories for harmonic transport}

The instantaneous average energy for a harmonically driven transport mode can be calculated from Eqs. (14) and (16):

$$
\begin{aligned}
& \left\langle\psi_{n}(t)|H(t)| \psi_{n}(t)\right\rangle \\
& \quad=\hbar \omega_{0}\left(n+\frac{1}{2}\right)+\frac{m}{2} \dot{q}_{c}^{2}+\frac{1}{2} m \omega_{0}^{2}\left(q_{c}-q_{0}\right)^{2} .
\end{aligned}
$$

Moreover, the instantaneous average potential energy is $\langle V(t)\rangle=\frac{\hbar \omega_{0}}{2}\left(n+\frac{1}{2}\right)+E_{P}$. The first, "internal" contribution remains constant for each $n$, and the second term has the simple form of a potential energy for a classical particle, $E_{P} \equiv \frac{1}{2} m \omega_{0}^{2}\left(q_{c}-q_{0}\right)^{2}$. Its time average, using relation (15) between $q_{0}$ and $q_{c}$, takes the form

$$
\overline{E_{P}}=\frac{m}{2 t_{f} \omega_{0}^{2}} \int_{0}^{t_{f}} \ddot{q}_{c}^{2} d t .
$$

We can use a generalized Euler-Lagrange equation $d^{4} q_{c} / d t^{4}=$ 0 to minimize this integral subject to four boundary conditions [39], the ones for $q_{c}$ and $\dot{q}_{c}$ in Eqs. (21) and (22). This results in a "quasioptimal" classical trajectory:

$$
q_{c}=d\left(3 s^{2}-2 s^{3}\right) .
$$

Whereas Eq. (34) does not satisfy the six boundary conditions (21) and (22), it provides, in any case, a lower bound for the time average of $E_{P}$, as the set of functions satisfying the six conditions is smaller than the one satisfying four of them. Substituting Eq. (34) into Eq. (33), one gets the desired lower bound,

$$
\overline{E_{P}} \geqslant \frac{6 m d^{2}}{t_{f}^{4} \omega_{0}^{2}} .
$$

This bound describes the relevant dependences, as shown by numerical comparisons with actual time-averaged energies for polynomial trajectories, Eq. (23) [see Fig. 4(a)], and sets a rather strong $t_{f}^{-4}$ scaling, compared with the milder dependence on $t_{f}^{-2}$ of the time-averaged transient energy in invariant-based, inverse-engineered expansions [40].

As for the variance $(\Delta H)^{2} \equiv\left\langle\psi_{n}\left|H^{2}\right| \psi_{n}\right\rangle-\left\langle\psi_{n}|H| \psi_{n}\right\rangle^{2}$ for the $n$th transport mode, it takes, after a somewhat lengthy calculation, a simple form:

$$
(\Delta H)^{2}=2 \hbar \omega_{0}(n+1 / 2)\left[\frac{1}{2} m \omega_{0}^{2}\left(q_{c}-q_{0}\right)^{2}+\frac{1}{2} m \dot{q}_{c}^{2}\right] .
$$
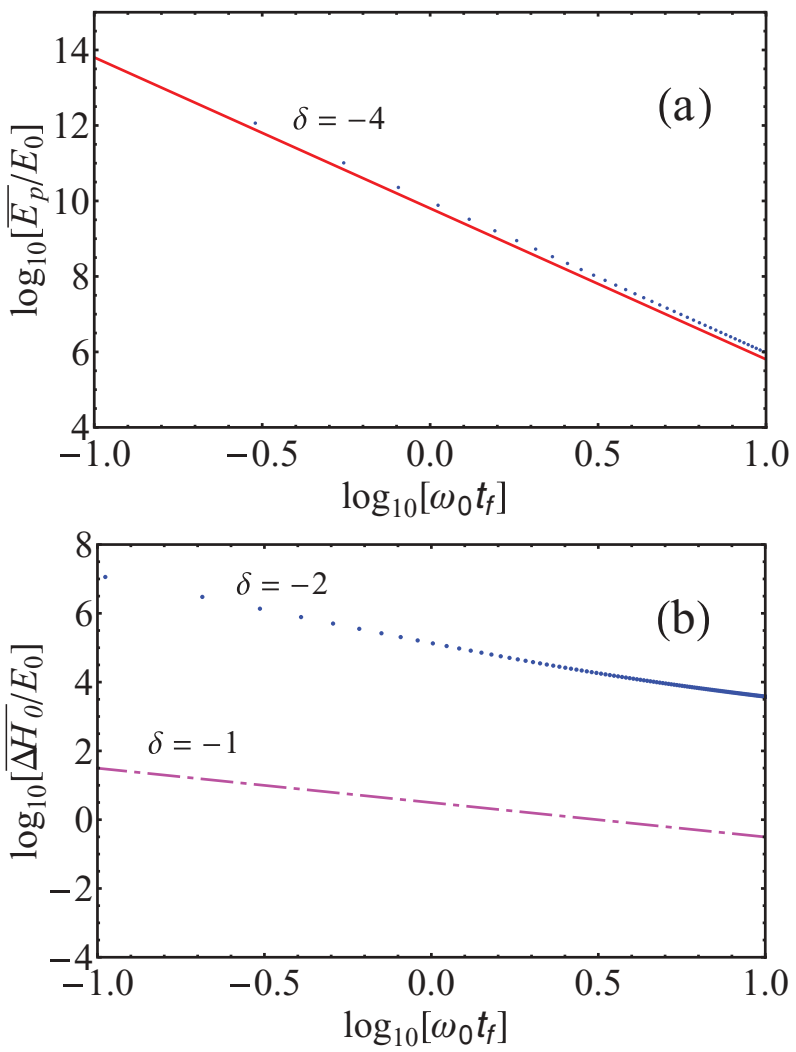

FIG. 4. (Color online) Dependences of time-averaged energies on $t_{f}$. The $\delta$ is the asymptotic exponent of $t_{f}$. Parameters are $\omega_{0}=$ $2 \pi \times 8 \mathrm{~Hz}, E_{0}=\hbar \omega_{0} / 2, d=2.25 \mathrm{~mm}$, and rubidium- 87 atoms that begin and end in the ground vibrational state. (a) Bound (35) (solid red line) and time average of $E_{P}$ for a polynomial trajectory (dotted blue line). (b) AA bound (dot-dashed magenta line) and time average of $\Delta H$ for a polynomial trajectory (dotted blue line).

Using again a Euler-Lagrange equation, we find the lower bound $\overline{(\Delta H)^{2}}>12 \hbar(n+1 / 2) m d^{2} / \omega_{0} t_{f}^{4}$ for its time average. This does not establish a lower bound for the average of the standard deviation $\overline{\Delta H}$ but agrees with the scaling with $t_{f}$ that we observe numerically as $t_{f} \rightarrow 0, \overline{\Delta H} \propto t_{f}^{-2}$; see Fig. 4(b). This should be contrasted with the AharonovAnandan (AA) relation [41] $\overline{\Delta H} \geqslant h / 4 t_{f}$, which is applied to transport among orthogonal states. (The general expression for ground-state to ground-state transport allowing for nonorthogonal initial and final states $[40,42]$ is $\overline{\Delta H} t_{f} \geqslant$ $\hbar \arccos \left[\exp \left(-m \omega_{0} d^{2} / 4 \hbar\right)\right]$, which tends to the result for orthogonal states when $d \gg\left(4 \hbar / m \omega_{0}\right)^{1 / 2}$.) As it occurs for harmonic trap expansions [40], it is certainly correct as a bound, but it does not describe the dependences found for the averaged standard deviation for fast processes ( small $t_{f}$ ).

\section{B. Mean-value theorem}

The mean-value theorem (MVT) sets another useful bound since it applies to the instantaneous values rather than to a time average. The argument may be applied to $q_{c}$ in case (i) or to $q_{0}$ in case (ii), so we shall formulate it in terms of a generic $x$, assumed to be continuous in $\left[0, t_{f}\right]$ and differentiable in $\left(0, t_{f}\right)$ 


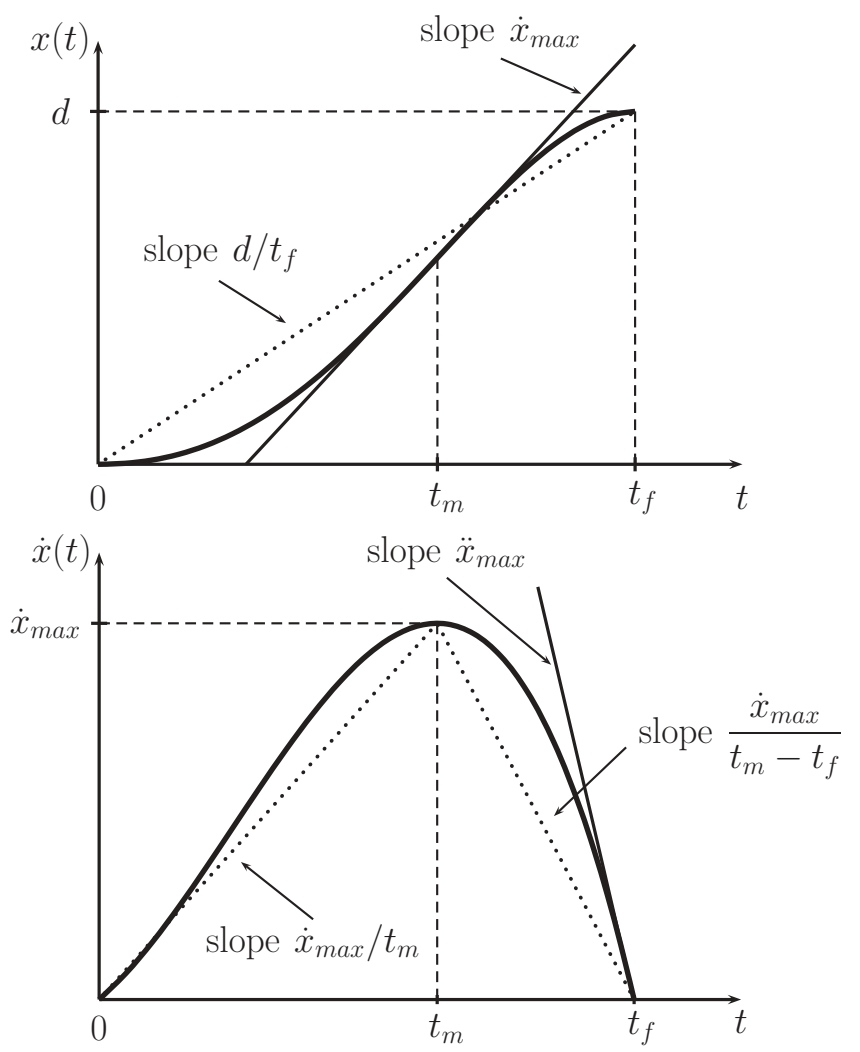

FIG. 5. Graphical representation of the lower bound of Eq. (37) for (top) the maximum velocity $\dot{x}_{\max }$ and (bottom) the maximum acceleration $|\ddot{x}|_{\max }$ of Eqs. (38) and (39).

and such that $x(0)=\dot{x}(0)=\dot{x}\left(t_{f}\right)=0$ and $x\left(t_{f}\right)=d$. The maximum of its time derivative must be

$$
\dot{x}_{\max } \geqslant d / t_{f}
$$

at some point $t_{m}$ in $\left(0, t_{f}\right)$; see Fig. 5 . We can now use that point to divide $\left[0, t_{f}\right]$ into two segments and apply the MVT again, now to the derivative. In the first segment the derivative goes from 0 to $\dot{x}_{\max }$, so

$$
|\ddot{x}|_{\max } \geqslant d /\left(t_{f} t_{m}\right) .
$$

Similarly, in the second segment,

$$
|\ddot{x}|_{\max } \geqslant d /\left[t_{f}\left(t_{f}-t_{m}\right)\right]
$$

and for the whole interval, $|\ddot{x}|_{\max } \geqslant d /\left[t_{f} \min \left(t_{m}, t_{f}-t_{m}\right)\right]$. Irrespective of the location of the point $t_{m}, \min \left(t_{m}, t_{f}-t_{m}\right) \leqslant$ $t_{f} / 2$. We can thus set a lower bound for the (absolute value of) the maximum of the second derivative,

$$
|\ddot{x}|_{\max } \geqslant 2 d / t_{f}^{2} \text {. }
$$

When $x=q_{c}$, case (i), Eq. (40) gives a bound for the instantaneous quasipotential energy:

$$
E_{P} \geqslant 2 m\left(\frac{d}{\omega t_{f}^{2}}\right)^{2} .
$$

In this case, however, we get a tighter lower bound directly from the time average (35).
With $x=q_{0}$, case (ii), we get a lower bound for the maximum trap acceleration. In particular, the accelerations of compensating forces, typically limited by experimental constraints such as gradient magnetic fields or Stark electric fields, should at the very least reach the value $2 d / t_{f}^{2}$.

\section{DISCUSSION}

We have applied the Lewis-Riesenfeld method [28] for quadratic-in- $p$ invariants [33] combined with inverse engineering of trap trajectories to design fast and faithful atomic transport. The limitations have been quantified, and relations to other approaches that can be included in this framework have been pointed out. Another approach to accelerate adiabatic processes has been recently proposed by Berry [43] and can also be applied formally to transport. The central idea is to construct a Hamiltonian that drives the system exactly along the adiabatic approximation defined for some reference time-dependent Hamiltonian $H_{0}(t)$ without transitions among the instantaneous eigenstates of $H_{0}$. For an arbitrary, rigid trap potential moving from 0 to $d$ along a path $q_{0}(t)$, the eigenvectors of the instantaneous (reference) Hamiltonian $H_{0}=p^{2} / 2 m+U\left(q-q_{0}\right)$ are simply displaced from the original location,

$$
|n(t)\rangle=e^{-i p q_{0}(t) / \hbar}|n(0)\rangle .
$$

The transitionless driving Hamiltonian $H_{0}+i \hbar \sum_{n}|\partial n\rangle\langle n|$ becomes, in this case, $H_{0}+p \dot{q}_{0}$; compare with Eq. (20). Note the freedom to choose $H_{0}$. It could even be suppressed during transport: the simple Hamiltonian $p \dot{q}_{0}$ would also keep the same populations (of any $H_{0}$ ) without generating dynamical phases $e^{-i E_{n} t}$ for each eigenvalue. This is a rather intuitive result since the corresponding propagator is nothing but the displacement operator $e^{-i p q_{0}(t) / \hbar}$. This approach thus provides a formal solution to fast and faithful transport, but the practical realization of a $\dot{q}_{0} p$ Hamiltonian term remains an open question.

With respect to the general framework embraced by Eqs. (1)-(11), the studied cases (i) and (ii) are very relevant but not exhaustive. For $U=0$, the most general case occurs when transport is accompanied by expansions and contractions, so that the time dependence of $\omega(t)$ and $\rho(t)$ has to be considered if the invariant-based inverse engineering method is applied.

For harmonic trap expansions or contractions in the gravity field ( $q$ becomes a vertical coordinate), $F=-m g$, and the center of the trap suffers a time-dependent translation $q_{0}=-2 g / \omega^{2}$. Again, $\alpha$ in Eq. (3) may be interpreted as a classical trajectory, now of a time-dependent harmonic oscillator subjected to the gravity field,

$$
\ddot{q}_{c}+\omega^{2}(t) \quad q_{c}=-g .
$$

This is admittedly not a proper transport problem, but its formal treatment is the same and has recently been implemented experimentally [44] and also for Bose-Einstein condensates [45].

As for further extensions or open questions of the invariant approach, one may investigate the use of more complex invariants that are not restricted to being quadratic in $p$ [46], in particular, to tackle anharmonic transport. In the frame of quadratic-in- $p$ invariants, anharmonic traps can be dealt 
with by a compensating force [case (ii)], but this force might be difficult to implement for large accelerations. If $F=0$, $\omega=0$, and $U(q-\alpha) \neq 0$ in Eq. (1), $\alpha$ should be the trap trajectory $q_{0}$, which is only consistent with the auxiliary equation $\ddot{\alpha}=0$ [see Eq. (3)] for constant-velocity trajectories which are incompatible with the boundary conditions (29). A way out, to be explored, may be to use the invariants to implement minimization algorithms of the final vibrational excitation.

Other research avenues from here include the analysis and optimization of transport for specific, commonly found potentials, such as periodic walking waves considering entangled states that correlate internal and external degrees of freedom (see, e.g., [47]) or the combination of invariant-based inverse engineering with optimal control theory [48]. Finally, further work will be devoted to understanding and mitigating the effects of noise, for which the inversion method is intrinsically robust [24], and of atom-atom interactions. Tonks-Girardeau gases can be treated similarly to single particles, and for Bose-Einstein condensates, scaling techniques may be used, as in $[45,49]$.

\section{ACKNOWLEDGMENTS}

We thank T. Lahaye and M. Berry for useful discussions. We also acknowledge the kind hospitality of the Max Planck Institute for the Physics of Complex Systems in Dresden and funding from the Basque government (Grant No. IT472-10), Ministerio de Ciencia e Innovación (Grant No. FIS2009-12773-C02-01), the Agence Nationale de la Recherche (Grant No. ANR-09-BLAN-0134-01), and the Région Midi-Pyrénées. E.T. and S.I. acknowledge financial support from the Basque government (Grant Nos. BFI08.151 and BFI09.39). X.C. acknowledges support from the Juan de la Cierva Programme and the National Natural Science Foundation of China (Grant No. 60806041).

\section{APPENDIX A: DISPLACEMENT UNITARY TRANSFORMATION}

Consider the following (time-dependent) position and momentum displacement unitary operator:

$$
\mathcal{U}=e^{i p q_{0}(t) / \hbar} e^{-i m \dot{q}_{0}(t) q / \hbar}
$$

We could also use variants of $\mathcal{U}$ with different orderings without affecting the final result. Starting from the Schrödinger equation,

$$
i \hbar \partial_{t}|\psi\rangle=H|\psi\rangle,
$$

where [as in Eq. (20)] $H=p^{2} / 2 m+U\left(q-q_{0}\right)-m q \ddot{q}_{0}$, the corresponding equation for $|\Phi\rangle=\mathcal{U}|\psi\rangle$ is

$$
\begin{aligned}
i \hbar \partial_{t}|\Phi\rangle & =\mathcal{U} H \mathcal{U}^{\dagger}|\Phi\rangle+i \hbar\left(\partial_{t} \mathcal{U}\right) U^{\dagger}|\Phi\rangle \\
& =\left[\frac{p^{2}}{2 m}+U(q)+\frac{m \dot{q}_{0}^{2}}{2}\right]|\Phi\rangle .
\end{aligned}
$$

Any stationary state in this "trap frame" will remain so in spite of the trap motion thanks to the compensating effect of the term $-m q \ddot{q}_{0}$ in $H$.

\section{APPENDIX B : PERTURBATION THEORY ANALYSIS OF THE EFFECT OF ANHARMONICITY}

In this Appendix we shall use perturbation theory to determine the effect of small anharmonicities using inverse or bang-bang trap trajectories. For concreteness, we start from the "cigar trap" potential associated with a Gaussian beam with a moving focus,

$$
V(\Delta, r)=-V_{0} e^{-2 r^{2} / w^{2}(\Delta)} \frac{1}{1+\frac{\Delta^{2}}{x_{R}^{2}}},
$$

where $r$ and $\Delta$ are radial and longitudinal coordinates, $\Delta=q-q_{0}(t)$

$$
w(\Delta)=w_{0} \sqrt{1+\left(\frac{\Delta}{x_{R}}\right)^{2}}
$$

is the spot size, $x_{R}=\pi w_{0}^{2} / \lambda$ is the Rayleigh length, and $w_{0}$ is the waist.

For a tight radial confinement we may ignore the radial coordinate and set $r=0$. The resulting longitudinal potential can be expanded around the minimum. Retaining the first correction to the harmonic term and ignoring the constant, we split the Hamiltonian, considering the quartic term as a perturbation,

$$
\begin{gathered}
H=H_{0}+V_{1}=\frac{p^{2}}{2 m}+V_{0} \frac{\left[q-q_{0}(t)\right]^{2}}{x_{R}^{2}}-V_{0} \frac{\left[q-q_{0}(t)\right]^{4}}{x_{R}^{4}}, \\
V_{1}=-V_{0} \frac{\left[q-q_{0}(t)\right]^{4}}{x_{R}^{4}},
\end{gathered}
$$

where $V_{0}=m \omega_{0}^{2} x_{R}^{2} / 2$. Using time-dependent perturbation theory, we calculate the overlap $\left\langle\psi\left(t_{f}\right) \mid \tilde{\psi}\left(t_{f}\right)\right\rangle$ between the state evolving with the harmonic oscillator $|\psi(t)\rangle$ and the perturbed state $|\tilde{\psi}(t)\rangle$ at the final time $t=t_{f} \cdot|\psi(t)\rangle$ is chosen as the transport mode (16), with (21) satisfied.

We approximate the perturbed state in first order as

$$
\begin{aligned}
|\tilde{\psi}(t)\rangle= & U_{0}(t, 0)|\tilde{\psi}(0)\rangle \\
& -\frac{i}{h} \int_{0}^{t} d t^{\prime} U_{0}\left(t, t^{\prime}\right) V\left(t^{\prime}\right) U_{0}\left(t^{\prime}, 0\right)|\tilde{\psi}(0)\rangle,
\end{aligned}
$$

where

$$
U_{0}(t, 0)=\exp \left(-\frac{i}{\hbar} \int_{0}^{t} d t^{\prime} H_{0}\left(t^{\prime}\right)\right)
$$

So

$$
\begin{aligned}
& \left\langle\psi\left(t_{f}\right) \mid \tilde{\psi}\left(t_{f}\right)\right\rangle \\
& =\left\langle\psi\left(t_{f}\right)\left|U_{0}\left(t_{f}, 0\right)\right| \tilde{\psi}(0)\right\rangle \\
& \quad-\frac{i}{\hbar} \int_{0}^{t_{f}} d t^{\prime}\left\langle\psi\left(t_{f}\right)\left|U_{0}\left(t_{f}, t^{\prime}\right) V\left(t^{\prime}\right) U_{0}\left(t^{\prime}, 0\right)\right| \tilde{\psi}(0)\right\rangle .
\end{aligned}
$$

At $t=0$ the initial state is also an eigenstate of the harmonic oscillator, $|\tilde{\psi}(0)\rangle=|\psi(0)\rangle$, so the first term on the right-hand side of Eq. (B6) is 1. Using the transport modes in Eq. (16), we calculate the bracket term in Eq. (B6),

$$
\begin{aligned}
& \left\langle\psi\left(t_{f}\right)\left|U_{0}\left(t_{f}, t^{\prime}\right) V\left(t^{\prime}\right) U_{0}\left(t^{\prime}, 0\right)\right| \tilde{\psi}(0)\right\rangle \\
& =\frac{-V_{0}}{x_{R}^{4}}\left\langle\psi\left(t^{\prime}\right)\left|\left[q-q_{0}\left(t^{\prime}\right)\right]^{4}\right| \psi\left(t^{\prime}\right)\right\rangle .
\end{aligned}
$$


Performing the time integral, we arrive, in first order, at

$$
\left\langle\psi\left(t_{f}\right) \mid \tilde{\psi}\left(t_{f}\right)\right\rangle=1-\frac{i}{\hbar} \mathcal{F} .
$$

The generic $\mathcal{F}$ becomes $\mathcal{F}_{\text {bb }}$ when substituting the functions for the bang-bang method described in Sec. IV for $q_{0}$ and $q_{c}$; see Eqs. (30) and (31).

$$
\begin{aligned}
\mathcal{F}_{\mathrm{bb}}= & \frac{2^{-(n+2)} V_{0}(2 n) ! !}{x_{R}^{4} n !}\left\{\frac{\hbar^{2} t_{f}}{m^{2} \omega_{0}^{2}}[3+6 n(1+n)]+\frac{48 d^{2} \hbar(1+2 n)}{m t_{f}^{4} \omega_{0}^{6}}\left[10 \omega_{0} t_{f}-4 \omega_{0} t_{f} \cos \left(\frac{\omega_{0} t_{f}}{2}\right)-28 \sin \left(\frac{\omega_{0} t_{f}}{2}\right)\right.\right. \\
& \left.+12 \sin \left(\omega_{0} t_{f}\right)-4 \sin \left(\frac{3 \omega_{0} t_{f}}{2}\right)+\sin \left(2 \omega_{0} t_{f}\right)\right]+\frac{32 d^{4}}{3 t_{f}^{8} \omega_{0}^{9}}\left[144 \omega_{0} t_{f}\left(-9 \cos \left(\frac{\omega_{0} t_{f}}{2}\right)+\cos \left(\omega_{0} t_{f}\right)\right)\right. \\
& -6096 \sin \left(\frac{\omega_{0} t_{f}}{2}\right)+4632 \sin \left(\omega_{0} t_{f}\right)+4\left(393 \omega_{0} t_{f}-530 \sin \left(\frac{3 \omega_{0} t_{f}}{2}\right)+222 \sin \left(2 \omega_{0} t_{f}\right)\right. \\
& \left.\left.\left.-84 \sin \left(\frac{5 \omega_{0} t_{f}}{2}\right)+26 \sin \left(3 \omega_{0} t_{f}\right)-6 \sin \left(\frac{7 \omega_{0} t_{f}}{2}\right)\right)+3 \sin \left(4 \omega_{0} t_{f}\right)\right]\right\}
\end{aligned}
$$

When $t_{f} \rightarrow 0$, then $\mathcal{F}_{\mathrm{bb}} \rightarrow 0$, but this is not a very useful limit since the bang-bang procedure will not work for times smaller than $4 \pi / \omega_{0}$. As a more interesting case, consider the discrete final times $t_{f, N}=4 \pi N / \omega_{0}$ with $N \in \mathbb{N}$ [19]. Then $\mathcal{F}_{\text {bb }}$ takes the form

$$
\mathcal{F}_{\mathrm{bb}}=\frac{-2^{-(10+n)}(2 n) ! !}{N^{7} m \pi^{7} n ! \omega_{0} x_{R}^{2}}\left\{1536 N^{8} \hbar^{2}[1+2 n(1+n)] \pi^{8}+576 d^{2} N^{4} \hbar m(1+2 n) \pi^{4} \omega_{0}+35 d^{4} m^{2} \omega_{0}^{2}\right\} .
$$

In the limit $x_{R} \rightarrow \infty, \mathcal{F}_{\mathrm{bb}} \rightarrow 0$. Increasing the waist and keeping the other parameters constant, the potential is more harmonic. In contrast, as $x_{R} \rightarrow 0, \mathcal{F}_{\mathrm{bb}} \rightarrow-\infty$. Also, $\mathcal{F}_{\mathrm{bb}} \rightarrow-\infty$ when $\omega_{0} \rightarrow 0$ and $\omega_{0} \rightarrow \infty$.

If instead of the bang-bang functions we choose the inverse-engineered polynomial $q_{c}$ in Eq. (23) and the corresponding $q_{0}$, for arbitrary parameters and, in particular, an arbitrary $t_{f}, \mathcal{F}$ becomes

$$
\mathcal{F}_{\text {inv }}=\frac{-2^{-(n+2)} V_{0}(2 n) ! !}{x_{R}^{4} n !}\left[\frac{1728000 d^{4}}{1001 t_{f}^{7} \omega_{0}^{8}}+\frac{1440 d^{2} \hbar(1+2 n)}{7 m t_{f}^{3} \omega_{0}^{5}}+\frac{\hbar^{2}\left[3+6 n(1+n) t_{f}\right]}{m^{2} \omega_{0}^{2}}\right] .
$$

For the final times $t_{f, N}$,

$$
\mathcal{F}_{\text {inv }}=\frac{-2^{-(8+n)} 3(2 n) ! !}{1001 N^{7} m \pi^{7} n ! \omega_{0} x_{R}^{2}}\left\{128128 N^{8} \hbar^{2}[1+2 n(1+n)] \pi^{8}+34320 d^{2} N^{4} \hbar m(1+2 n) \pi^{4} \omega_{0}+1125 d^{4} m^{2} \omega_{0}^{2}\right\}
$$

Comparing the factors in (B10) and (B11), we see that $\left|\mathcal{F}_{\text {inv }}\right|<\left|\mathcal{F}_{\mathrm{bb}}\right|$ for $\omega_{0}>0$.

[1] M. A. Rowe et al., Quantum Inf. Comput. 4, 257 (2002).

[2] R. Reichle, D. Leibfried, R. B. Blakestad, J. Britton, J. D. Jost, E. Knill, C. Langer, R. Ozeri, S. Seidelin, and D. J. Wineland, Fortschr. Phys. 54, 666 (2006).

[3] Y. Miroschnychenko, W. Alt, I. Dotsenko, L. Förster, M. Khudaverdyan, D. Meschede, D. Schrader, and A. Rauschenbeutel, Nature (London) 442, 151 (2006).

[4] T. L. Gustavson, A. P. Chikkatur, A. E. Leanhardt, A. Görlitz, S. Gupta, D. E. Pritchard, and W. Ketterle, Phys. Rev. Lett. 88, 020401 (2001).

[5] H. J. Lewandowski, D. M. Harber, D. L. Whitaker, and E. A. Cornell, J. Low Temp. Phys. 132, 309 (2003).

[6] M. J. Pritchard, A. S. Arnold, S. L. Cornish, D. W. Hallwood, C. V. S. Pleasant, and I. G. Hughes, New J. Phys. 8, 309 (2006).

[7] G. R. Guthöhrlein, M. Keller, K. Hayasaka, W. Lange, and H. Walther, Nature (London) 414, 49 (2001).

[8] T. Calarco, E. A. Hinds, D. Jaksch, J. Schmiedmayer, J. I. Cirac, and P. Zoller, Phys. Rev. A 61, 022304 (2000).

[9] J. D. Prestage, R. L. Tjoelker, G. J. Dick, and L. Maleki, in Proceedings of the 1993 IEEE Frequency Control Symposium (IEEE, Piscataway, NJ, 1993), p. 144.
[10] S. Kuhr, W. Alt, D. Schrader, M. Müller, V. Gomer, and D. Meschede, Science 293, 278 (2001).

[11] S. Schmidt, J. G. Muga, and A. Ruschhaupt, Phys. Rev. A 80, 023406 (2009).

[12] X. Chen, J. G. Muga, A. del Campo, and A. Ruschhaupt, Phys. Rev. A 80, 063421 (2009).

[13] W. Hänsel, J. Reichel, P. Hommelhoff, and T. W. Hänsch, Phys. Rev. Lett. 86, 608 (2001).

[14] W. Hänsel, P. Hommelhoff, T. W. Hänsch, and J. Reichel, Nature (London) 413, 498 (2001).

[15] S. Kuhr, W. Alt, D. Schrader, I. Dotsenko, Y. Miroshnychenko, W. Rosenfeld, M. Khudaverdyan, V. Gomer, A. Rauschenbeutel, and D. Meschede, Phys. Rev. Lett. 91, 213002 (2003).

[16] D. Schrader, S. Kuhr, W. Alt, M. Müller, V. Gomer, and D. Meschede, Appl. Phys. B 73, 819 (2001).

[17] M. Greiner, I. Bloch, T. W. Hänsch, and T. Esslinger, Phys. Rev. A 63, 031401 (2001).

[18] T. Lahaye, G. Reinaudi, Z. Wang, A. Couvert, and D. Guéry-Odelin, Phys. Rev. A 74, 033622 (2006).

[19] A. Couvert, T. Kawalec, G. Reinaudi, and D. Guéry-Odelin, Europhys. Lett. 83, 13001 (2008). 
[20] S. Schmid, G. Thalhammer, K. Winkler, F. Lang, and J. H. Denschlag, New J. Phys. 8, 159 (2006).

[21] G. Huber, T. Deuschle, W. Schnitzler, R. Reichle, K. Singer, and F. Schmidt-Kaler, New J. Phys. 10, 013004 (2008).

[22] D. R. Crick, S. Donnellan, S. Ananthamurthy, R. C. Thompson, and D. M. Segal, Rev. Sci. Instrum. 81, 013111 (2010).

[23] R. B. Blakestad, C. Ospelkaus, A. P. VanDevender, J. M. Amini, J. Britton, D. Leibfried, and D. J. Wineland, Phys. Rev. Lett. 102, 153002 (2009).

[24] M. Murphy, L. Jiang, N. Khaneja, and T. Calarco, Phys. Rev. A 79, 020301(R) (2009).

[25] S. Masuda and K. Nakamura, Proc. R. Soc. A 466, 1135 (2010).

[26] D. Chen, H. Zhang, X. Xu, T. Li, and Y. Wang, Appl. Phys. Lett. 96, 134103 (2010).

[27] X. Chen, A. Ruschhaupt, S. Schmidt, A. del Campo, D. Guéry-Odelin, and J. G. Muga, Phys. Rev. Lett. 104, 063002 (2010).

[28] H. R. Lewis and W. B. Riesenfeld, J. Math. Phys. 10, 1458 (1969).

[29] V. P. Ermakov, Univ. Izv. Kiev. 20, 1 (1880).

[30] H. R. Lewis and P. G. Leach, J. Math. Phys. 23, 2371 (1982).

[31] P. G. L. Leach, J. Math. Phys. 18, 1608 (1977).

[32] P. G. L. Leach, J. Math. Phys. 18, 1902 (1977).

[33] A. K. Dhara and S. W. Lawande, J. Phys. A 17, 2423 (1984).

[34] E. Narevicius et al., New J. Phys. 9, 96 (2007).
[35] M. Raizen, Science 324, 1403 (2009).

[36] H. L. Bethlem, G. Berden, and G. Meijer, Phys. Rev. Lett. 83, 1558 (1999).

[37] S. Y. T. van de Meerakker, P. H. M. Smeets, N. Vanhaecke, R. T. Jongma, and G. Meijer, Phys. Rev. Lett. 94, 023004 (2005).

[38] B. C. Sawyer, B. L. Lev, E. R. Hudson, B. K. Stuhl, M. Lara, J. L. Bohn, and J. Ye, Phys. Rev. Lett. 98, 253002 (2007).

[39] I. M. Gelfand and S. V. Fomin, Calculus of Variations (Prentice-Hall, Englewood Chiffs, New Jersey, 1963).

[40] X. Chen and J. G. Muga, Phys. Rev. A 82, 053403 (2010).

[41] J. Anandan and Y. Aharonov, Phys. Rev. Lett. 65, 1697 (1990).

[42] A. K. Pati, Phys. Lett. A 262, 296 (1999).

[43] M. V. Berry, J. Phys. A 42, 365303 (2009).

[44] J. F. Schaff, X.-L. Song, P. Vignolo, and G. Labeyrie, Phys. Rev. A 82, 033430 (2010).

[45] J. F. Schaff, X. L. Song, P. Capuzzi, P. Vignolo, and G. Labeyrie, e-print arXiv: 1009.5868 .

[46] Y. H. Lee and J. Y. Riu, J. Korean Phys. Soc. 25, 469 (1992).

[47] M. J. McDonnell, J. P. Home, D. M. Lucas, G. Imreh, B. C. Keitch, D. J. Szwer, N. R. Thomas, S. C. Webster, D. N. Stacey, and A. M. Steane, Phys. Rev. Lett. 98, 063603 (2007).

[48] D. Stefanatos, J. Ruths, and Jr-Shin Li, Phys. Rev. A 82, 063422 (2010).

[49] J. G. Muga, X. Chen, A. Ruschhaupt, and D. Guéry-Odelin, J. Phys. B 42, 241001 (2009). 